RESEARCH ARTICLE

\author{
V. A. Artamonov • R. B. Mukhatov • R. Wisbauer
}

\title{
On the category of modules over some semisimple bialgebras
}

Received: 30 September 2010 / Accepted: 4 January 2011 / Published online: 24 March 2012

(C) The Author(s) 2012. This article is published with open access at Springerlink.com

\begin{abstract}
We study the tensor category of modules over a semisimple bialgebra $H$ under the assumption that irreducible $H$-modules of the same dimension $>1$ are isomorphic. We consider properties of Clebsch-Gordan coefficients showing multiplicities of occurrences of each irreducible $H$-module in a tensor product of irreducible ones. It is shown that, in general, these coefficients cannot have small values.
\end{abstract}

\section{Mathematics Subject Classification 16T10}
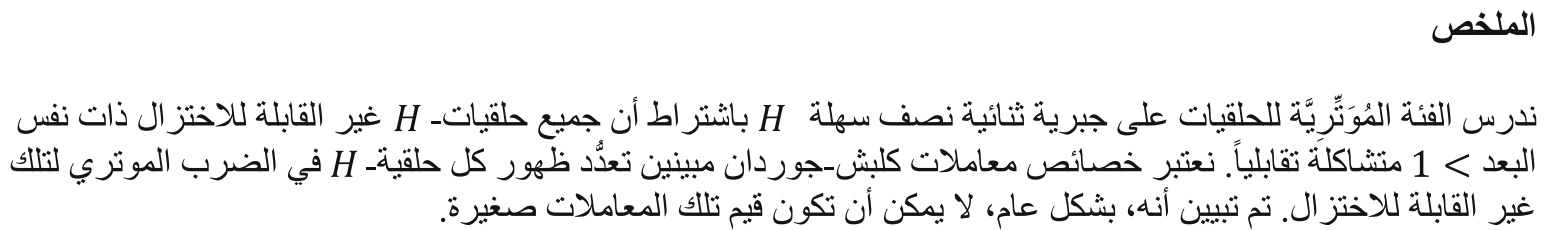

\section{Introduction}

Throughout the paper, the basic field $k$ is algebraically closed and $H$ is a finite dimensional $k$-bialgebra that is semisimple as an algebra. The restriction that $k$ is algebraically closed implies that any finite dimensional simple $k$-algebra is a full matrix algebra over $k$. We shall use the notations for bialgebras and Hopf algebras from $[4,5]$.

An element $g \in H$ is a group-like element if $\Delta(g)=g \otimes g$ and $\varepsilon(g)=1$. The set of all group-like elements $G(H)$ of a bialgebra $H$ is a multiplicative monoid. If $H$ is a Hopf algebra with an antipode $S$, then $G(H)$ is a group, where $g^{-1}=S(g)$ for any $g \in G(H)$.

The dual bialgebra $H^{*}$ has a natural pairing $\langle-,-\rangle: H^{*} \otimes H \rightarrow k$. The monoid $G=G\left(H^{*}\right)$ of group-like elements in $H^{*}$ consists just of algebra homomorphisms $H \rightarrow k$.

V. A. Artamonov $(\varangle) \cdot$ R. B. Mukhatov

Faculty of Mechanics and Mathematics, Department of Algebra,

Moscow State University, Moscow, Russia

E-mail: artamon@mech.math.msu.su

R. Wisbauer

Matematisches Institut,

H. Heine University Düsseldorf, Düsseldorf, Russia

E-mail: wisbauer@math.uni-duesseldorf.de 
A semisimple algebra $H$ is a direct sum of full matrix algebras over $k$. One-dimensional summands are in one-to-one correspondence with algebra homomorphisms $H \rightarrow k$. Hence, under our assumptions, $H$ as a $k$-algebra has a semisimple direct decomposition

$$
H=\left(\bigoplus_{g \in G} k e_{g}\right) \oplus\left(\bigoplus_{j=1}^{n} \operatorname{Mat}\left(d_{j}, k\right)\right),
$$

where $n, d_{j}$ are natural numbers and $\left\{e_{g}, g \in G\right\}$ is a system of central orthogonal idempotents in $H$ corresponding to the one-dimensional direct summands. For $h \in H$ and $g \in G$ we have $h e_{g}=e_{g} h=\langle g, h\rangle e_{g}$.

As in [1], we here deal with the case when

$$
1<d_{1}<d_{2}<\cdots<d_{n},
$$

which just means that irreducible $H$-modules of the same dimension $>1$ are isomorphic.

The main result of the paper [1] is the following:

Theorem 1.1 Let $H$ be a semisimple Hopf algebra with decomposition (1.1), $n \geqslant 1$, such that (1.2) holds. Suppose that at least one single matrix constituent is a Hopf ideal in $H$. Then it is the last summand $\operatorname{Mat}\left(d_{n}, k\right)$.

In the present paper, for a bialgebra $H$, we consider properties of the Clebsch-Gordan coefficients, that is, the multiplicities of occurrences of irreducible $H$-modules in semisimple decompositions of tensor products of irreducible ones. These play a substantial role in representation theory of groups and their applications to physics.

More general than in [1], we consider the case of a bialgebra $H$ not assuming that it is a Hopf algebra. In Theorem 4.5, under some restrictions on the Clebsch-Gordan coefficients, it is shown that $n \leqslant 2$ in (1.1). In Theorem 4.6, for the case $n=2$, we compare the number of one-dimensional summands in (1.1) and the sizes of matrix components. Further properties of Clebsch-Gordan coefficients are found in Theorem 4.7. In the last section we consider the comodule structure of $H$.

\section{Bialgebra structure of $H$ and $H^{*}$}

We consider comultiplication and counit in the bialgebra $H$ having as algebra a decomposition (1.1). The counit $\varepsilon: H \rightarrow k$ has the form

$$
\varepsilon(x)= \begin{cases}\delta_{g, 1}, & x=e_{g}, \\ 0, & x \in \operatorname{Mat}\left(d_{i}, k\right) .\end{cases}
$$

For each one-dimensional $H$-module $E_{g}=k e_{g}$ related to $g \in G$,

$$
h e_{g}=\langle g, h\rangle e_{g}, \quad h \in H .
$$

For further information on the bialgebra structure of $H$ some additional properties of the dual bialgebra $H^{*}$ are needed.

The semisimple bialgebra $H$ over an algebraically closed field $k$ has the decomposition (1.1). If char $k=0$ and $H$ is a Hopf algebra, then, by the Larson-Radford theorem [4, Theorem 7.4.6], the dual Hopf algebra $H^{*}$ is also semisimple. Recall that some additional information on semisimple Hopf algebras in positive characteristic can be found in [6].

Consider one of the main samples of bialgebras, namely a monoid algebra $F=k G$ of a finite monoid $G$. In this case $\Delta(g)=g \otimes g$ for any $g \in G$. It means that $G$ is the monoid of group-like elements of $F$.

It is well-known that the dual bialgebra $F^{*}$ is a direct sum of one-dimensional ideals $\oplus_{g \in G} k e_{g}$. Here $\left\{e_{g} \mid g \in G\right\}$ is the dual base for the base $\{g \mid g \in G\}$ of $F$. In particular, $F^{*}$ is semisimple.

However, its dual bialgebra $F^{* *}=F$ is not necessarily semisimple. For example, take the three-element commutative monoid $G=\{1, a, b\}$ with the identity element 1 such that $a b=b^{2}=a^{2}=b$. Then the one-dimensional space $k(a-b)$ in the monoid algebra $F=k G$ is annihilated by $a, b$. Hence it is a nilpotent ideal and the monoid algebra $k G$ is not semisimple.

We shall now expand these structural observations to the case of the bialgebra $H$ from (1.1). 
Consider in each matrix component $\operatorname{Mat}\left(d_{i}, k\right)$, the non-degenerated symmetric bilinear form

$$
\langle x, y\rangle=\operatorname{tr}\left(x \cdot{ }^{t} y\right) .
$$

In the case of a Hopf algebra we consider the form $\langle x, y\rangle=\operatorname{tr}(x \cdot S(y))$ where $S$ is the antipode [3]. We shall prove results from [3, Section 3] on Hopf algebras for the bialgebra case.

Using the form (2.3), we can identify the space Mat $\left(d_{i}, k\right)$ with its dual space. Then the base of Mat $\left(d_{i}, k\right)$ consisting of matrix units $E_{\alpha \beta}^{(i)}, \alpha, \beta=1, \ldots, d_{i}$, is self-dual, namely

$$
\left\langle E_{\alpha \beta}^{(i)}, E_{\gamma \tau}^{(i)}\right\rangle=\operatorname{tr}\left(E_{\alpha \beta}^{(i)} E_{\tau \gamma}^{(i)}\right)=\delta_{\beta \tau} \operatorname{tr}\left(E_{\alpha \gamma}^{(i)}\right)=\delta_{\beta \tau} \delta_{\alpha \gamma}
$$

Thus, as a vector space, $H^{*}$ has a direct decomposition

$$
H^{*}=k G \oplus \operatorname{Mat}\left(d_{1}, k\right) \oplus \cdots \oplus \operatorname{Mat}\left(d_{n}, k\right) .
$$

The counit $\varepsilon^{*}$ in $H^{*}$ is defined as $\varepsilon(f)=f(1)$ for any $f \in H^{*}$, where 1 is the unit of $H$, and $1=$ $\sum_{g \in G} e_{g}+E^{(1)}+\cdots+E^{(n)} \in H$. Direct calculations, as in [3], show $\varepsilon(g)=1, \quad \varepsilon(x)=\operatorname{tr}(x)$, if $g \in G, x \in \operatorname{Mat}\left(d_{i}, k\right)$. The comultiplication $\Delta^{*}$ in $H^{*}$ is defined by $\left\langle\Delta^{*}(f), a \otimes b\right\rangle=\langle f, a b\rangle$, for all $a, b \in H$.

Proposition 2.1 The following conditions are satisfied:

(i) For $g \in G, \Delta^{*}(g)=g \otimes g$.

(ii) For the matrix unit $E_{\alpha \beta}^{(i)}$ from the $i$-th matrix component,

$$
\Delta^{*}\left(E_{\alpha \beta}^{(i)}\right)=\sum_{\gamma} E_{\alpha \gamma}^{(i)} \otimes E_{\gamma \beta}^{(i)}
$$

Proof Let

$$
a=\sum_{g \in G} \tau_{g} g+\sum_{\substack{i=1, \ldots, n ; \\ \alpha \beta=1, \ldots, d_{i}}} E_{\alpha \beta}^{(i)} a_{\alpha \beta}^{(i)}, \quad b=\sum_{g \in G} \xi_{g} g+\sum_{\substack{i=1, \ldots, n ; \\ \gamma, \lambda=1, \ldots, d_{i}}} E_{\gamma \lambda}^{(i)} b_{\gamma \lambda}^{(i)},
$$

where $\tau_{g}, \xi_{g}, a_{\alpha \beta}^{(i)}, b_{\gamma \lambda}^{(i)} \in k$. Then

$$
a b=\sum_{g \in G} \tau_{g} \xi_{g} g+\sum_{\substack{i=1, \ldots, n ; \\ \alpha, \lambda=1, \ldots, d_{i}}} E_{\alpha \lambda}^{(i)}\left(\sum_{\beta=1}^{d_{i}} a_{\alpha \beta}^{(i)} b_{\beta \lambda}^{(i)}\right)
$$

So, if $g \in G$, then $\left\langle\Delta^{*}(g), a \otimes b\right\rangle=\langle g, a b\rangle=\tau_{g} \xi_{g}=\langle g, a\rangle\langle g, b\rangle=\langle g \otimes g, a \otimes b\rangle$, hence $\Delta^{*}(g)=g \otimes g$. Now

$$
\begin{aligned}
\left\langle\Delta^{*}\left(E_{\alpha \lambda}^{(i)}\right), a \otimes b\right\rangle & =\left\langle E_{\alpha \lambda}^{(i)}, a b\right\rangle=\sum_{\beta=1}^{d_{i}} a_{\alpha \beta}^{(i)} b_{\beta \lambda}^{(i)}=\sum_{\beta=1}^{d_{i}}\left\langle E_{\alpha \beta}^{(i)}, a\right\rangle\left\langle E_{\beta \lambda}^{(i)}, b\right\rangle \\
& =\left\langle\sum_{\beta=1}^{d_{i}} E_{\alpha \beta}^{(i)} \otimes E_{\beta \lambda}^{(i)}, a \otimes b\right\rangle,
\end{aligned}
$$

and this means $\Delta^{*}\left(E_{\alpha \lambda}^{(i)}\right)=\sum_{\beta=1}^{d_{i}} E_{\alpha \beta}^{(i)} \otimes E_{\beta \lambda}^{(i)}$.

Proposition 2.2 If $p, q \in G$, then $p * q=p q$. Suppose that $H$ is a Hopf algebra. If $x \in \operatorname{Mat}\left(d_{i}, k\right)$, then $p * x=p \rightarrow x, x * p=x<p$. 
Proof Suppose that $a$ is from (2.4). Then by (2.6)

$$
\langle p * q, a\rangle=\sum_{g, h, f \in G, h f=g} \tau_{g}\left\langle p, e_{h}\right\rangle\left\langle q, e_{f}\right\rangle=\tau_{p q}=\langle p q, a\rangle
$$

and therefore $p * q=p q$.

In the case of Hopf algebras we can prove the last formulas as in [3].

Now we shall consider some new properties of the bialgebra $H$ from (1.1). The bialgebra $H$ is a left and right $H^{*}$-module algebra with respect to actions $f \rightarrow x, x<f$ of $f \in H^{*}$ on $x \in H$, [5, Example 4.1.10], that is, for $\Delta(x)=\sum_{x} x_{(1)} \otimes x_{(2)}$,

$$
f \rightarrow x=\sum_{x} x_{(1)}\left\langle f, x_{(2)}\right\rangle, \quad x<f=\sum_{x}\left\langle f, x_{(1)}\right\rangle x_{(2)} .
$$

For $f \in G$, the maps $f \rightarrow, \angle f$ are algebra endomorphisms of $H$ preserving the identity element 1 of $H$, and $1=\sum_{f \in G} e_{f}+\sum_{i \geqslant 1} E^{(i)}$, where $E^{(i)}$ is the identity matrix of Mat $\left(d_{i}, k\right)$.

As shown in [2, Propposition 1.3, Corollary 1.2],

$$
\begin{aligned}
\Delta\left(e_{g}\right) & =\sum_{p, q \in G, p q=g} e_{p} \otimes e_{q}+\sum_{i=1}^{n} \mathcal{D}_{g, i} ; \\
\Delta(x) & =\sum_{g \in G}\left((g-x) \otimes e_{g}+e_{g} \otimes(x<g)\right)+\sum_{i, j=1}^{n} \Delta_{i j}^{t}(x),
\end{aligned}
$$

where $\mathcal{D}_{g, i} \in \operatorname{Mat}\left(d_{i}, k\right)^{\otimes 2}$ and, $\Delta_{i j}^{t}(x) \in \operatorname{Mat}\left(d_{i}, k\right) \otimes \operatorname{Mat}\left(d_{j}, k\right)$, for $i, j=1, \ldots, n$.

With respect to the natural pairing $\langle-,-\rangle$, the elements $g \in G \subset H^{*}$ are dual to the elements $e_{g}, g \in G$, and each matrix component is annihilated by elements of $G$.

Proposition 2.3 (1) The element $e_{1}$ is the left and the right integral in $H$.

(2) For $g, f \in G, g \rightarrow e_{f}$ is equal either to zero or to the sum of all $e_{p}, p \in G$, such that $p g=f$.

(3) An element $g \in G$ is invertible if and only if $g \rightarrow e_{1} \neq 0$.

(4) For $g \in G$,

$$
g \rightarrow\left(\sum_{f \in G} e_{f}\right)=\sum_{f \in G} e_{f}, \quad g \rightarrow\left(\sum_{i} E^{(i)}\right)=\left(\sum_{i} E^{(i)}\right),
$$

where the $E^{(i)}$ denote the identity matrix in $\operatorname{Mat}\left(d_{i}, k\right)$.

Proof (1) For $h \in H, h e_{1}=\langle 1, h\rangle e_{1}=\varepsilon(h) e_{1}$ by (2.1) and (2.2).

(2) Using the first equation in (2.6), we obtain

$$
g \rightarrow e_{f}=\sum_{p, q \in G, p q=f} e_{p}\left\langle g, e_{q}\right\rangle=\sum_{p \in G, p g=f} e_{p} .
$$

(3) By (2), the element $g \rightarrow e_{1} \neq 0$ if and only if there exists an element $p \in G$ such that $p g=1$. It means that $p=g^{-1}$.

(4) Let $g \in G$. The map $h \mapsto(g \rightarrow h)$ is an algebra endomorphism of $H$ preserving the unit element $1=\sum_{f \in G} e_{f}+\sum_{i \geqslant 1} E^{(i)}$, where $E^{(i)}$ is the identity matrix of Mat $\left(d_{i}, k\right)$. Each full matrix algebra $\operatorname{Mat}\left(d_{i}, k\right)$ is simple and therefore it is mapping either to zero or injectively into $H$. Hence we obtain the required equality by (2).

Theorem 2.4 Let $\alpha$ be a unit preserving endomorphism of the semisimple algebra $R=\oplus_{i=1}^{n} \operatorname{Mat}\left(d_{i}, k\right)$, where $1<d_{1}<d_{2}<\cdots<d_{n}$. Suppose that each integer $d_{j}$ is not a linear combination of $d_{1}, \ldots, d_{j-1}$ with non-negative integer coefficients. Then $\alpha$ is an automorphism of $R$ preserving each matrix component. 
Proof We shall proceed by induction on $n$. If $n=1$, then $\alpha$ is an endomorphism of the full matrix algebra preserving the unit element. Hence $\alpha$ is injective and therefore it is surjective.

Suppose that the theorem is proved for $n-1$. Since $d_{n}>d_{j}$ for any $j<n$ we can conclude that $\operatorname{Mat}\left(d_{n}, k\right)$ is stable under $\alpha$. By induction, $\alpha$ induces an automorphism on $R / \operatorname{Mat}\left(d_{n}, k\right)$. So without loss of generality we can assume that $\alpha$ is identical modulo $\operatorname{Mat}\left(d_{n}, k\right)$. It means that if $x \in \operatorname{Mat}\left(d_{j}, k\right), j<n$, then $\alpha(x)=x+\beta_{j}(x)$, where $\beta_{j}: \operatorname{Mat}\left(d_{j}, k\right) \rightarrow \operatorname{Mat}\left(d_{n}, k\right)$ is an algebra homomorphism, not necessarily preserving the unit element.

Suppose first that $\alpha\left(E^{(n)}\right) \neq 0$. Then $\alpha$ induces an automorphism of Mat $\left(d_{n}, k\right)$ and therefore $\alpha\left(E^{(n)}\right)=$ $E^{(n)}$. If $x \in \operatorname{Mat}\left(d_{j}, k\right), j<n$ then $x E^{(n)}=0$ in $R$ and therefore

$$
0=\alpha(x) \alpha\left(E^{(n)}\right)=\left(x+\beta_{j}(x)\right) E^{(n)}=\beta_{j}(x) E^{(n)}=\beta_{j}(x) .
$$

Hence, in this case, $\alpha$ is an automorphism and the proof is complete.

Suppose that Mat $\left(d_{n}, k\right)$ is contained in the kernel of $\alpha$. Then $E^{(n)}=\beta_{1}\left(E^{(1)}\right)+\cdots+\beta_{n-1}\left(E^{(n-1)}\right)$ because $\alpha$ preserves the unit element of $R$. Note that $\beta_{i}(x) \beta_{j}(y)=0$ if $i \neq j$, so the elements $\beta_{1}\left(E^{(1)}\right), \ldots, \beta_{n-1}\left(E^{(n-1)}\right)$ form an orthogonal system of idempotents of sizes $t_{1}, \ldots, t_{n-1}$, respectively, and therefore $t_{1}+\cdots+t_{n-1}=d_{n}$.

By the Noether-Skolem and centralizer theorems, we can conclude that $\operatorname{Mat}\left(t_{j}, k\right) \simeq \beta_{j}\left(\operatorname{Mat}\left(d_{j}, k\right)\right) \otimes$ $\operatorname{Mat}\left(s_{j}, k\right)$ for some non-negative integer $s_{j}$. Hence $t_{j}=d_{j} s_{j}$ and therefore $d_{n}=t_{1}+\cdots+t_{n-1}=d_{1} s_{1}+$ $\cdots+d_{n-1} s_{n-1}$, a contradiction.

Note that the restriction on the numbers in Theorem 2.4 is satisfied if, for each $j$, the greatest common divisor of $d_{1}, \ldots d_{j}$ is smaller than the greatest common divisor of $d_{1}, \ldots, d_{j-1}$.

\section{The category of modules}

Let $H$ be, as above, a semisimple bialgebra with direct sum decomposition (1.1) such that (1.2) is satisfied. In what follows we shall in addition assume that either $G$ is a group or $d_{1}, \ldots, d_{n}$ are as in Theorem 2.4. In both cases, for each $g \in G$, the map $g \rightarrow$ induces an algebra automorphism of every matrix component in (1.1).

The tensor product $M \otimes N$ of two left $H$-modules $M, N$ is again a left $H$-module by putting, for $h \in H$ and $\Delta(h)=\sum_{h} h_{(1)} \otimes h_{(2)}$,

$$
h(x \otimes y):=\sum_{h} h_{(1)} x \otimes h_{(2)} y, \quad x \in M, \quad y \in N .
$$

Let $M_{i}$ be the irreducible $H$-module associated with matrix component $\operatorname{Mat}\left(d_{i}, k\right)$. The module $M_{i}$ is annihilated by each element $e_{g}, g \in G$, and by any $\operatorname{Mat}\left(d_{j}, k\right), j \neq i$.

Note that if $h \in \operatorname{Mat}\left(d_{i}, k\right)$ and $x \in M_{p}, y \in M_{q}$, then by (3.1) we have

$$
h(x \otimes y)=\Delta_{p q}^{i}(h) \cdot(x \otimes y),
$$

where $\Delta_{p q}^{i}(h) \cdot(x \otimes y)$ is the componentwise action on the tensor product.

As in [1, Formula (9), Lemma 3.1] we can prove:

Proposition 3.1 Let $h \in H, g \in G$ and $\mathcal{D}_{g, i}$ from (2.6). If $x, y \in M_{i}$ then $h\left(\mathcal{D}_{g, i} \cdot(x \otimes y)\right)=\langle g, h\rangle \mathcal{D}_{g, i} \cdot(x \otimes y)$ and $\mathcal{D}_{g, i}^{2}=\mathcal{D}_{g, i}$.

Proof We have

$$
\begin{aligned}
& h\left(\mathcal{D}_{g, i} \cdot(x \otimes y)\right)=\left(\Delta(h) \mathcal{D}_{g, i}\right) \cdot(x \otimes y) \\
& =\left(\Delta(h) \Delta\left(e_{g}\right)\right) \cdot(x \otimes y)=\Delta\left(h e_{g}\right) \cdot(x \otimes y)=\langle g, h\rangle \mathcal{D}_{g, i} \cdot(x \otimes y) .
\end{aligned}
$$

The last statement holds because $e_{g}$ is an idempotent.

The next fact is well known for Hopf algebras [1]. In virtue of Theorem 2.3 it holds for bialgebras $H$ satisfying the above restrictions. 
Proposition 3.2 Let $H$ be a bialgebra with a direct decomposition (1.1) such that (1.2) holds. Suppose M to be an irreducible $H$-module, $\operatorname{dim} M>1$. Let $E_{g}$ be the one-dimensional $H$-module associated with an element $g \in G$. Then $M \otimes E_{g}$ and $E_{g} \otimes M$ are irreducible $H$-modules and

$$
M \otimes E_{g} \simeq E_{g} \otimes M \simeq M .
$$

For any square matrix $X$ denote its transpose by ${ }^{t} X$. Let $M_{i}$ be as above the irreducible $H$-module of dimension $d_{i}$ Then the dual space $M_{i}^{*}=\operatorname{Hom}_{k}\left(M_{i}, k\right)$ is a left $H$-module. In fact, let $f \in M_{i}^{*}, h \in \operatorname{Mat}\left(d_{i}, k\right)$ and $x \in M_{i}$. Put $\langle h \cdot f, x\rangle=\left\langle f,{ }^{t} h \cdot x\right\rangle$. Then for $h_{1}, h_{2} \in \operatorname{Mat}\left(d_{i}, k\right)$,

$$
\begin{aligned}
\left\langle h_{1} h_{2} \cdot f, x\right\rangle=\left\langle f,{ }^{t}\left(h_{1} h_{2}\right) \cdot x\right\rangle & =\left\langle f,{ }^{t} h_{2}{ }^{t} h_{1} \cdot x\right\rangle \\
& =\left\langle h_{2} \cdot f,{ }^{t} h_{1} \cdot x\right\rangle=\left\langle h_{1} \cdot\left(h_{2} \cdot f\right), x\right\rangle .
\end{aligned}
$$

Using [4, Lemma 7.5.10, p. 322] as in [1, Proposition 1.7], we obtain

Proposition 3.3 Let $M_{i}, M_{j}$ be irreducible left $H$-modules of dimensions $>1$. Then $\operatorname{dim} \operatorname{Hom}_{H}\left(M_{i} \otimes\right.$ $\left.M_{j}, E_{\varepsilon}\right)=\delta_{i j}$

Proposition 3.4 Denote by $A$ the direct sum $\oplus_{g \in G} E_{g}$ of all one-dimensional $H$-modules $E_{g}, g \in G$. Then there is a direct sum decomposition

$$
M_{i} \otimes M_{j}=\delta_{i j} A \oplus\left(\oplus_{t=1}^{n} m_{i j}^{t} M_{t}\right)
$$

where $m_{i j}^{t}=\operatorname{dim}_{k} \operatorname{Hom}_{H}\left(M_{i} \otimes M_{j}, M_{t}\right) \geqslant 0$. In particular,

$$
\begin{aligned}
\operatorname{dim}\left(M_{i} \otimes M_{j}\right) & =d_{i} d_{j}=\delta_{i j}|G|+\sum_{t=1}^{n} m_{i j}^{t} d_{t} \\
& =\operatorname{dim}\left(\delta_{i j} A \oplus\left(\oplus_{t=1}^{n} m_{i j}^{t} M_{t}\right)\right)
\end{aligned}
$$

and $|G| \leqslant d_{1}^{2}$.

Proposition 3.4 generalizes [1, Corollary 1.8, Theorem 1.9] from Hopf algebras to the case of bialgebras with the mentioned properties.

Using Proposition 3.1, we can prove as in [1, Lemma 3.1]:

Corollary 3.5 Let $\mu: E_{g} \rightarrow M_{i} \otimes M_{i}$ be an embedding of $H$-modules from Proposition 3.4. Then $\mu\left(E_{g}\right)=$ $\mathcal{D}_{g, i}\left(M_{i} \otimes M_{i}\right)$.

The next affirmation follows from associativity of tensor products of $H$-modules.

Theorem 3.6 ([1]) The multiplicities $m_{i j}^{t}$ defined in Proposition 3.4 satisfy the Eq. (3.4) and the equations

$$
m_{i j}^{s}=m_{j s}^{i}, \quad \delta_{i j} \delta_{l s}|G|+\sum_{t=1}^{n} m_{i j}^{t} m_{t s}^{l}=\delta_{j s} \delta_{l i}|G|+\sum_{t=1}^{n} m_{j s}^{t} m_{i t}^{l},
$$

for all $i, j, s, l=1, \ldots, n$. In particular, $m_{i j}^{s}=m_{j s}^{i}=m_{s i}^{j}$ and

$$
\delta_{i j} \delta_{l s}|G|+\sum_{t=1}^{n} m_{t i}^{j} m_{t s}^{l}=\delta_{j s} \delta_{l i}|G|+\sum_{t=1}^{n} m_{s t}^{j} m_{i t}^{l} .
$$

If $i, j, p=1, \ldots, n$, then $m_{i j}^{p} \leqslant d_{\min (i, j, p)}$.

Furthermore, if $H$ is a Hopf algebra, then $m_{p q}^{i}=m_{q p}^{i}$ for all $i, p, q=1, \ldots, n$, that is, $M_{i} \otimes M_{j} \simeq$ $M_{j} \otimes M_{i}$ for all $i, j=1, \ldots, n$. 
Denote by $R_{t}, 1 \leqslant t \leqslant n$, the square matrix of size $n$ whose $(i, j)$ th entry is equal to $m_{i j}^{t}$. Then $R_{r}$ is a non-negative integer matrix. By Theorem 3.6, each matrix $R_{t}$ is symmetric. Now the equality (3.4) and the statement of Theorem 3.6 can be rewritten as

$$
\begin{aligned}
{\left[{ }^{t} R_{j}, R_{l}\right] } & =|G|\left(E_{l j}-E_{j l}\right) \\
\sum_{t} d_{t} R_{t} & =\left(\begin{array}{c}
d_{1} \\
\vdots \\
d_{n}
\end{array}\right)\left(d_{1} \ldots d_{n}\right)-|G| E_{n},
\end{aligned}
$$

where $E_{n}$ and $E_{l j}$ are the identity matrix and the matrix units of size $n$. If $H$ is a Hopf algebra, then each matrix $R_{i}$ is symmetric.

For later use consider the case $n=2$. In view of Theorem 3.6 put

$$
a=m_{11}^{1}, b=m_{12}^{1}=m_{21}^{1}=m_{11}^{2}, c=m_{22}^{1}=m_{12}^{2}=m_{21}^{2}, d=m_{22}^{2},
$$

which all are non-negative integers. Then

$$
R_{1}=\left(\begin{array}{ll}
a & b \\
b & c
\end{array}\right), \quad R_{2}=\left(\begin{array}{ll}
b & c \\
c & d
\end{array}\right)
$$

Now the first equation in (3.5) can be rewritten as

$$
b^{2}+c^{2}-a c-b d=|G|
$$

and the second equation in (3.5) as

$$
\begin{aligned}
& d_{1} a+d_{2} b=d_{1}^{2}-|G|, \\
& d_{1} b+d_{2} c=d_{1} d_{2}, \\
& d_{1} c+d_{2} d=d_{2}^{2}-|G| .
\end{aligned}
$$

\section{Properties of coefficients}

In this section we shall consider properties of the Clebsch-Gordan coefficients $m_{i j}^{t}$ in the decomposition (3.3) for a bialgebra $H$ with decomposition (1.1) and with additional properties from Sect. 3.

Proposition 4.1 Let $H$ be a bialgebra as above and $M_{p}, M_{q}$ irreducible $H$-modules of dimensions greater than 1, such that $M_{p} \otimes M_{q}$ and $M_{q} \otimes M_{p}$ are irreducible H-modules. Then the order of the monoid $G$ is equal to 1 . If $H$ is a Hopf algebra then $M_{p} \otimes M_{q} \simeq M_{q} \otimes M_{p}$.

Proof Suppose the $H$-module $M_{p} \otimes M_{q}$ is irreducible for some indices $p, q=1, \ldots, n$. Then $p \neq q$ by Proposition 3.4. So $M_{p} \otimes M_{q} \simeq M_{i}$ for some index $i=1, \ldots, n$. It means that $m_{p q}^{i}=1=m_{i q}^{p}$. Note that the indices $i, p, q$ are distinct because $d_{i}=d_{p} d_{q}>d_{p}, d_{q}$. In particular $n \geqslant 3$.

Associativity of the tensor product of modules yields by Theorem 3.6, since $m_{p q}^{i}=1=m_{q i}^{p}$,

$$
\begin{aligned}
M_{p} \otimes M_{q} \otimes M_{q} & \simeq M_{p} \otimes\left(A \oplus\left(\oplus_{t} m_{q q}^{t} M_{t}\right)\right) \\
& \simeq\left(M_{p} \otimes A\right) \oplus\left[\oplus_{t} m_{q q}^{t}\left(M_{p} \otimes M_{t}\right)\right] \\
& \simeq|G| M_{p} \oplus m_{q q}^{p} A \oplus\left[\left(\oplus_{t, s} m_{q q}^{t} m_{p t}^{s} M_{s}\right)\right] ; \\
M_{p} \otimes M_{q} \otimes M_{q} & \simeq M_{i} \otimes M_{q}=M_{p} \oplus\left[\oplus_{t \neq p} m_{i q}^{t} M_{t}\right] .
\end{aligned}
$$

Comparing coefficients in $M_{p}$, we obtain $|G|+\sum_{t} m_{q q}^{t} m_{p t}^{p}=1$. Hence $|G|=1$.

Consider other cases when tensor products of some irreducible $H$-modules have similar almost trivial decompositions. 
Proposition 4.2 Let $1 \leqslant i \neq j \leqslant n$. Suppose that there exists a unique index $t$ such that $m_{i j}^{t} \geqslant 1$. Then $t \geqslant \max (i, j)$.

Proof By the assumption,

$$
M_{i} \otimes M_{j} \simeq m_{i j}^{t} M_{t}
$$

Theorem 3.6 and (4.1) imply

$$
m_{i j}^{t}=\frac{\operatorname{dim} M_{i} \cdot \operatorname{dim} M_{j}}{\operatorname{dim} M_{t}}=\frac{d_{i} \cdot d_{j}}{d_{t}} \leqslant d_{\min (i, j, t)} \leqslant d_{i} .
$$

Hence $d_{j} \leqslant d_{t}$ which means that $j \leqslant t$. Similarly $i \leqslant t$.

Proposition 4.3 Suppose that (4.1) holds for some $t \neq i$ and

$$
M_{t} \otimes M_{i} \simeq m_{t i}^{t^{\prime}} M_{t^{\prime}},
$$

for some index $t^{\prime}$. Then $t=t^{\prime}=j>i$ and $m_{i j}^{t}=m_{j i}^{t}=d_{i}$.

Proof By Proposition 4.2 and the assumption, $t \geqslant \max (i, j)$. Since $t>i$ we can apply Theorem 3.6 and get $m_{i j}^{t}=m_{t i}^{j}>0$. So $t^{\prime}=j$ by the assumption and $M_{t} \otimes M_{i} \simeq m_{t i}^{j} M_{j}$. Applying Proposition 4.2 we obtain $j \geqslant \max (t, i)=t \geqslant j$ and therefore $t=j>i$ because $j \neq i$. Comparing dimensions we complete the proof.

Proposition 4.4 Let $i$ be an index with the property: for every index $j \neq i$, there exists a unique index $t$ such $m_{i j}^{t}>0$ and if $t \neq i$, then also (4.2) holds for some index $t^{\prime}$. Then:

(1) if $j \neq i$, then $M_{i} \otimes M_{j} \simeq d_{\min (i, j)} M_{\max (i, j)}$;

(2) $M_{i} \otimes M_{i} \simeq A \oplus d_{1} M_{1} \oplus \cdots \oplus d_{i-1} M_{i-1} \oplus m_{i i}^{i} M_{i}$;

(3) $d_{i}^{2}=|G|+d_{1}^{2}+\cdots+d_{i-1}^{2}+m_{i i}^{i} d_{i}$; in particular, if $i=1$, then the order of the monoid $G$ is divisible by $d_{1}$;

(4) $\Delta\left(\operatorname{Mat}\left(d_{i}, k\right)\right) \subseteq H \otimes \operatorname{Mat}\left(d_{i}, k\right)+\operatorname{Mat}\left(d_{i}, k\right) \otimes H+\left(\sum_{j \geqslant i} \operatorname{Mat}\left(d_{j}, k\right)^{\otimes 2}\right)$.

Proof (1) Suppose that $j>i$. Then $t \geqslant \max (i, j)=j>i$ by Proposition 4.2 and $t=j, m_{i j}^{j}=m_{j j}^{i}=d_{i}$.

If $j<i$, then, by Proposition 4.3, the case $t \neq i$ is impossible. Hence $j<i$ implies $t=i$ and $m_{i j}^{i}=d_{j}$. So in all cases (1) is proved. Moreover, for any $j \neq i$,

$$
m_{i j}^{s}= \begin{cases}d_{\min (i, j)}, & s=\max (i, j) ; \\ 0, & \text { otherwise. }\end{cases}
$$

(2) By Theorem 3.6, there is an $H$-module decomposition

$$
M_{i} \otimes M_{i} \simeq A \oplus\left(\oplus_{j} m_{i i}^{j} M_{s}\right) .
$$

Note that $m_{i i}^{j}=m_{i j}^{i}$. Hence, by (4.3), the inequality $m_{i i}^{j}>0$ implies $i=\max (i, j)>j$ and in this case $m_{i j}^{i}=d_{j}$. Hence we obtain the required decomposition of $M_{i} \otimes M_{i}$.

(3) Comparing dimensions in the decomposition from (2) we can obtain the required equality. In particular if $i=1$, then $d_{1}^{2}=|G|+m_{11}^{1} d_{1}$ and therefore $|G|$ is divisible by $d_{1}$.

(4) Take any indices $p, q=1, \ldots, n$ such that $\Delta_{p q}^{i} \neq 0$ in (2.6). Combining (2.6), (3.2) and Proposition 4.4, properties (1), (2), we see that $\operatorname{Mat}\left(d_{i}, k\right)$ annihilates $M_{p} \otimes M_{q}$ if either $i \neq \max (p, q)$ where $p \neq q$ or $p=q<i$. By (3.2) it means that (4) is satisfied.

Theorem 4.5 Let $H$ be a bialgebra with decomposition (1.1) such that (1.2) is satisfied and either $G$ is a group or $d_{1}, \ldots, d_{n}$ are as in Theorem 2.4. Suppose that $H$ satisfy the assumptions of Proposition 4.4 for some index $i$. If $i=1$, then $J=\oplus_{j} \geqslant 2 \operatorname{Mat}\left(d_{j}, k\right)$ is a bi-ideal in $H$. If $i=n$, then Mat $\left(d_{i}, k\right)$ is a bi-ideal of $H$. 
Proof Let $i=1$ and $\Delta_{p q}^{j} \neq 0$ for some $j \geqslant 2$ where either $p=1$ or $q=1$. The case $p=q=1$ is impossible by Proposition 4.4, (1) and (2). Hence either $p$ or $q$ is greater than 1. Hence $J$ is a bi-ideal.

Suppose that $i=n$ and $\Delta_{p q}^{n} \neq 0$ for some $p, q$. If either $p<n$ or $q<n$, then, by Proposition 4.4, (1), $n=\max (p, q)$ and therefore either $p=n$ or $q=n$. In both cases,

$$
\Delta\left(\operatorname{Mat}\left(d_{n}, k\right)\right) \subseteq H \otimes \operatorname{Mat}\left(d_{n}, k\right) \oplus \operatorname{Mat}\left(d_{n}, k\right) \otimes H .
$$

Theorem 4.6 Let $H$ be a Hopf algebra with decomposition (1.1). If the number $n$ of full matrix algebras of size $>1$ in (1.1) is equal to 2 , then the greatest common divisor $D$ of sizes $d_{1}, d_{2}$ of matrices is greater than 1. The order of the group $G$ is divisible by $D$.

Proof As it is noticed in [7] the order $|G|$ of the group $G$ divides $d_{1}^{2}$ and $d_{2}^{2}$. Suppose that $d_{1}, d_{2}$ are coprime. Using the notations (3.6), we see in the second equation in (3.9) that $b$ is divisible by $d_{2}$ and $c$ is divisible by $d_{1}$, namely $b=d_{2} u_{1}, c=d_{1} u_{2}$ for some non-negative integers $u_{1}, u_{2}$. So this equation can be rewritten as $u_{1}+u_{2}=1$. It follows immediately that there is an alternative,

$$
\text { either } u_{1}=1, u_{2}=0 \text {, or } u_{1}=0, u_{2}=1 \text {. }
$$

Suppose first that $u_{1}=1, u_{2}=0$. Then $b=d_{2}, c=0$ and the first equation in (3.9) has the form $d_{1} a+d_{2}^{2}=d_{1}^{2}-|G|$. This is impossible because $d_{2}>d_{1}$ but the left hand side is greater or equal to $d_{2}^{2}$ while the right hand side is smaller than $d_{1}^{2}$.

Suppose now that $u_{1}=0, u_{2}=1$. Then $b=0, c=d_{1}$ and the first equation in (3.9) has the form $d_{1} a=d_{1}^{2}-1$ which is impossible since $d_{1}>1$.

Theorem 4.7 Let $H$ be a semisimple bialgebra with decomposition (1.1) where $n \geqslant 2$. Then $m_{n-1, n}^{t} \geqslant 2$ for some index $t=1, \ldots, n$ in (3.3).

Proof Suppose that $m_{n-1, n}^{t} \leqslant 1$ for all $t=1, \ldots, n$. Then, in equation (3.3), we have $d_{n-1} d_{n} \leqslant d_{1}+\cdots+d_{n}$. Dividing by $d_{n}$ we get by (1.2),

$$
d_{n-1} \leqslant \frac{d_{1}}{d_{n}}+\cdots+\frac{d_{n-1}}{d_{n}}+1<n
$$

On the other hand, (1.2) implies that $d_{i} \geqslant i+1$ for any $i$ and in particular $d_{n-1}>n$, a contradiction.

\section{The category of $(H, H)$-bimodules}

Let, as above $H$, be the semisimple bialgebra with decomposition (1.1). By (3.1) the comultiplication $\Delta$ : $H \rightarrow H \otimes H$ is also a homomorphism of $(H, H)$-bimodules. So it is interesting to look at the structure of $(H, H)$-bimodules.

Note that any $(H, H)$-bimodule can be considered as a left module over $H \otimes H^{o p}$ where $H^{o p}$ is defined on the same vector space as $H$ by the new multiplication $x \cdot y=y x$. Clearly $H^{o p}$ is a semisimple algebra with a similar decomposition (1.1). Its irreducible modules are dual modules $E_{g}^{*}, g \in G$, and $M_{1}^{*}, \ldots, M_{n}^{*}$. The action of $h \in H^{o p}$ on $E_{g}^{*}$ and on $M_{i}^{*}$ is the following. If $f \in E_{g}^{*}$ then $\left\langle f h, e_{g}\right\rangle=\langle g, h\rangle\left\langle f, e_{g}\right\rangle$. If $f \in M_{i}^{*}$ and $x \in M_{i}$ then $\langle f h, x\rangle=\langle f, h x\rangle$. By Proposition 1.5 [1], each $M_{i}^{*}$ is an irreducible $H^{o p}$-module.

Now $H^{o p}$ is a bialgebra with comultiplication $\Delta^{o p}=\Delta$ and a counit $\varepsilon^{o p}=\varepsilon$.

Consider the bialgebra $H \otimes H^{o p}$. It is a semisimple bialgebra whose simple ideals are tensor products of simple ideals of $H$ and of $H^{o p}$. It means that irreducible $H \otimes H^{o p}$-modules are just tensor products

$$
E_{g} \otimes E_{f}^{*}, \quad E_{g} \otimes M_{i}^{*}, \quad M_{j} \otimes E_{g}^{*}, \quad M_{i} \otimes M_{j}^{*}, \quad f, g \in G .
$$

The one-dimensional bimodule $E_{g} \otimes E_{f}^{*}$ has a base $e_{g} \otimes e_{f}$ such that

$$
h\left(e_{g} \otimes e_{f}\right) r=\langle g, h\rangle\langle f, r\rangle\left(e_{g} \otimes e_{f}\right),
$$

for all $h, r \in H$. 
By Proposition 3.2 and Proposition 1.5 [1], the bimodule $E_{g} \otimes M_{i}^{*}$ can be identified with $M_{i}$ where $h x r=\langle g, h\rangle \cdot{ }^{t} r \cdot x$ for all $h, r \in H$ and $x \in M_{i}$.

The bimodule $M_{j} \otimes E_{g}^{*}$ can be identified with $M_{i}$ where $h x r=h x\langle g, r\rangle$ for all $h, r \in H$ and $x \in M_{i}$.

Finally, the bimodule $M_{i} \otimes M_{j}^{*}$ is identified with $M_{i} \otimes M_{j}$ where $h x r=h x \cdot{ }^{t} r$ for all $h, r \in H$ and $x \in M_{i}$.

Acknowledgments The authors are grateful to the referee for valuable remarks and suggestions. Research partially supported by grants DFG, RFBR 09-01-00058, 09-01-90416-Ucr-f-a.

Open Access This article is distributed under the terms of the Creative Commons Attribution License which permits any use, distribution, and reproduction in any medium, provided the original author(s) and the source are credited.

\section{References}

1. Artamonov, V.A.: On semisimple Hopf algebras with few representations of dimension greater than one. Révista de la Unión Matemática Argentina, 51(2), 89-103 (2010)

2. Artamonov, V.A.: On semisimple finite dimensional Hopf algebras. Math. Sbornik. 198(9), 3-28 (2007)

3. Artamonov, V.A.; Chubarov, I.A.: Dual algebras of some semisimple finite dimensional Hopf algebras. In: Modules and Comodules, Trends in Mathematics. Birkhauser, Basel, pp. 65-85 (2008)

4. Dăscălescu, S.; Năstăsescu, C.; Raianu, Ş.: Hopf Algebras. An Introduction. Marcel Dekker, Inc, Basel (2000)

5. Montgomery, S.: Hopf Algebras and Their Actions on Rings. Regional Conf. Ser. Math. American Mathematical Society, Providence (1993)

6. Masuoka, A.: Semisimplicity criteria for irreducible Hopf algebras in positive characteristic. Proc. Am. Math. Soc. 137(6), 1925-1932 (2009)

7. Natale, S.; Plavnik, J.Y.: On fusion categories with few irreducible degrees. ArXiv: 1103.23402 [mathQA] (2011) 